\title{
Cardiology Predictor: cardiology interpretations for medical diagnosing
}

\section{Nallaperuma \\ K. Lokuge}

Contact e-mail address: sachidin@gmail.com

eHealth Sri Lanka 2010,1(suppl.1):S33

DOI: http://dx.doi.org/10.4038/sljbmi.v1i0.3592

Only the Abstract is available

\begin{abstract}
Cardiology is an exceptionally complicated medical area where inaccurate and inefficient diagnosis could be fatal to a patient. Cardiology diagnosis is done by interpreting ECG reports and considering medical factors such as blood pressure, pulse rate, cholesterol level, and blood test reports. Moreover patient's history, gender and age are really important when diagnosing cardiovascular disease. Complicated ECG data are difficult to analyse as the text book pattern for a particular disease will not exist in the real world.

Therefore, medical practitioners who have not gone through a special training for ECG reading or inexperienced medical practitioners, find it a tedious task to interpret ECG and diagnose diseases. Doctors who are not cardiologists find it complicated to diagnose cardiologic abnormalities accurately. Nurses feel helpless when a doctor is not near while a heart patient is struggling for life. Meanwhile medical students find it extremely hard to study the cardiology subject with complicated patterns in the ECGs.

As a result, the level of accuracy and the efficiency in cardiovascular disease diagnosis is low and the 'Cardiology Predictor' is a software system which is capable of assisting medical practitioners to diagnose the diseases accurately. It takes in the ECG and other cardiac factors in order to output the cardiovascular disease. With the intention of accuracy and efficiency, ECG signals were used with digital signal processing for decrypting and feature extracting. Furthermore, artificial intelligence has to be used to diagnose cardiovascular disease due to the complexity embedded into it. Therefore, Artificial Neural Network was used to predict a cardiovascular disease. The average success rate of the system is 85.6 per cent based on the user evaluation and the domain experts confirmed that the system is most suitable for the emergency room where the expertise knowledge is lacking.
\end{abstract}

Keywords - Cardiology Predictor, cardiology interpretations, medical diagnosing 